\title{
Neural Network-Based Resistance Spot Welding Control and Quality Prediction
}

\author{
Nenad Ivezic, John D. Allen Jr., and Thomas Zacharia \\ Computer Science and Mathematics Division \\ Oak Ridge National Laboratory \\ Oak Ridge, TN 37831, USA
}

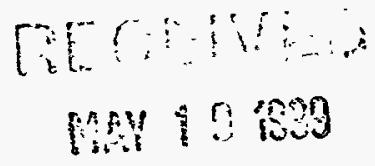

\begin{abstract}
(1)

This paper describes the development and evaluation of neural network-based systems for industrial resistance spot welding process control and weld quality assessment. The developed systems utilize recurrent neural networks for process control and both recurrent networks and static networks for quality prediction. The first section describes a system capable of both welding process control and real-time weld quality assessment. The second describes the development and evaluation of a static neural network-based weld quality assessment system that relied on experimental design to limit the influence of environmental variability. Relevant data analysis methods are also discussed. The weld classifier resulting from the analysis successfully balances predictive power and simplicity of interpretation. The results presented for both systems demonstrate clearly that neural networks can be employed to address two significant problems common to the resistance spot welding industry, control of the process itself, and non-destructive determination of resulting weld quality.
\end{abstract}

\section{INTRODUCTION}

Several factors may influence the quality of a forming resistance spot weld. Among the more important of these are failures in weld tip geometry, improper alignment of welder electrodes and metal surfaces to be joined, dirt and corrosion on the electrodes and/or metal surfaces, and uncompensated variations in AC supply voltage. Each of these may influence the principal variables (the welder output current and weld tip voltage drop) to which the weld control/quality assessment system is permitted to have access. Of primary importance is the signature defined by the temporal variations of these two quantities.

The first task of the work reported here was to develop a system capable of controlling the resistance spot welding process in real time. Realization of such a capability depended critically on development of methods for detecting, and compensating for, influential factors on a short enough time scale to support modulation of an evolving weld. The recurrent neural network system developed for this purpose is discussed in the next section.

The second task was to develop a system suitable for performing a posteriori weld quality assessment. In one approach, the recurrent neural network system developed as part of the first task was adapted to produce evolving evaluations of welding signatures and to extract from them a measure of weld quality. In an alternative approach, a static neural network was incorporated in an adaptive system that proved capable of determining weld quality by predicting key characteristics of the weld - nugget size and indentation - and by the subsequent mapping of these characteristics onto a pass/fail classification.

\section{EXPERIMENTS IN WELD EVOLUTION CAPTURE FOR PROCESS CONTROL AND QUALITY ASSESSMENT}

Critically important to the production of quality spot welds is the application of appropriate welding current for a time sufficient to ensure the formation of the weld nugget and short enough to avoid undesirable effects (e.g., excessive denting of the material surfaces and thinning of the weld region due to the prolonged application of electrode pressure). A reliable indicator of the onset of these and other processes is the expulsion of weld material that occurs when the forming weld nugget begins to exceed in volume that which can be effectively contained within the electrode boundaries. Welding current should be (indeed, should already have been) removed when this onset is 


\section{DISCLAIMER}

This report was prepared as an account of work sponsored by an agency of the United States Government. Neither the United States Government nor any agency thereof, nor any of their employees, make any warranty, express or implied, or assumes any legal liability or responsibility for the accuracy, completeness, or usefulness of any information, apparatus, product, or process disclosed, or represents that its use would not infringe privately owned rights. Reference herein to any specific commercial product, process, or service by trade name, trademark, manufacturer, or otherwise does not necessarily constitute or imply its endorsement, recommendation, or favoring by the United States Government or any agency thereof. The views and opinions of authors expressed herein do not necessarily state or reflect those of the United States Government or any agency thereof. 


\section{DISCLAIMER}

Portions of this document may be illegible in electronic image products. Images are produced from the best available original document. 
detected. In many spot welding control systems, this detection is effected by real-time analysis of derivatives of the weld resistance versus time characteristic of the welding process. Because any control function resulting from such an analysis must, of necessity, occur after the analysis has determined that expulsion is beginning, weld integrity may be irreversibly compromised before the welding process can be terminated.

\section{Real-Time Weld Evolution Prediction}

In preparation for the investigation of real-time prediction of weld characteristics, a commercial automotive spot welding apparatus and controller were installed in our laboratory and provided with a high-speed data acquisition system capable of acquiring weld data at speeds adequate for the purposes of developing a real-time process control system. Also included in the system was a "compensation coil" from which data could be derived for counteracting the inductive effects of the very large welding currents. Compensation data were also employed to support a sensing system that eliminated the necessity for making tip voltage measurements at the welding electrodes themselves, an important consideration in an industrial setting.

The first experiments with the laboratory welding apparatus were designed to demonstrate the feasibility of real-time prediction of dynamic resistance values during weld production. If such prediction proved possible and reliable, the prediction results could be trivially incorporated into a real-time weld-termination system and, with somewhat more effort, into a dynamic weld control system as well. The network(s) employed for the purpose were simple feedforward recurrent perceptrons enhanced with several sets of integrating shift-register input nodes through which the signals fed back from an intermediate layer were cycled with each presentation at the normal input nodes of new welding data. Each set of shift-register nodes was characterized by a unique time constant, the individual values being chosen to optimize the "historical" memory of the network. For these studies, the interval between measured welding data and the ensuing predicted values was limited to no more than three weld cycles. Figure 1 depicts the network predictions for weld resistance together with the observed resistance values for a series of 49 resistance spot welds of duration spanning a few to a few tens of weld cycles. Note that the welds were not obtained in succession, as the figure suggests. Rather, prediction results from the 49 welds were combined in a single data set to simplify subsequent analysis. In any case, the predicted values are so nearly identical to the subsequently observed values (which, for display and comparison purposes, were shifted into registry with the observed data) that the disparities are scarcely visible at the scale of the figure. Thus, it was concluded that, at the very least, dynamic control of the weld termination process could be effected with the so-far developed system.

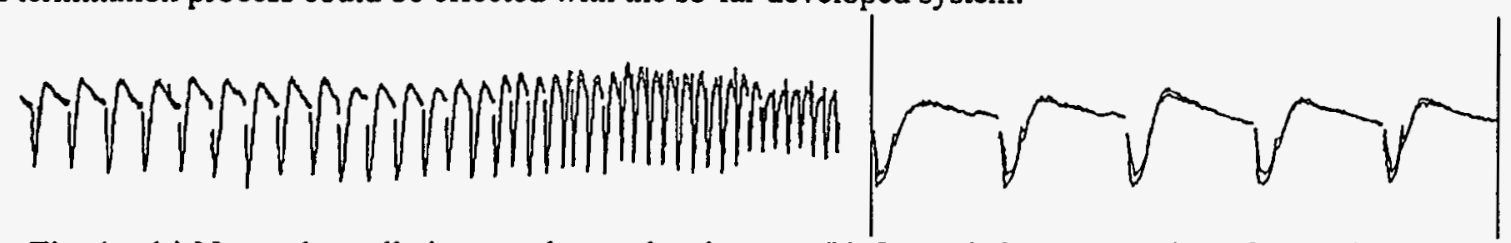

Fig. 1. (a) Network prediction vs. observed resistance; (b) Expanded representation of a previous figure

\section{WELD QUALITY ASSESSMENT WITH ATTRACTOR NEURAL NETWORKS}

Development of the attractor-based weld classification system represented a natural extension of the real-time dynamic weld-termination system described above. The method is suitable for application both in the context of real-time welding environments and in a posteriori assessment of previously acquired welding data.

Central to the attractor method is a novel dynamic network training mechanism deriving from considerations of two obvious aspects of the weld classification problem. It would be, for instance, entirely unreasonable to expect any system to determine weld quality on the basis of data representing only the first few weld cycles (or the middle few, or the last few, for that matter). On the other hand, it is reasonable to expect that the accuracy of a weld quality estimate should improve as more and more cycles of a particular weld event are completed. Thus, whatever a system might do in response to temporally evolving data for which a final "Good / Bad" rating was required should be done in a manner such that (1), very little response was given during the initial phase of data presentation (here, the first few weld cycles) and (2), a very stable response obtained during the final moments of data presentation (i.e., the last few weld cycles). This requirement was realized with an attractor network by imposing as the network training goal a signal having the form of a (suitably offset) square root of a half-sine, a function having relatively modest and 
slowly varying slopes at the two extrema and a much greater slope throughout a broad central region. During network training, welds known to be good were forced to define an output training function beginning at half full node output and following the square root of a half-sine function to maximum output at the end of the weld (whatever the number of weld half-cycles comprising the complete weld). Welds known to be bad were forced to define a training function beginning at half full output and following the square root of an inverted half-sine function to minimum output at the end of the weld (again, whatever the total number of weld half-cycles). These endpoints for "good" and "bad" welds defined two attractor basins for the network.

The network developed for the time-dependent classification task was very similar in form to the recurrent network described above and comprised an input layer, a single hidden layer, and an output layer. The input layer for a typical classification network included sixteen nodes devoted to the single-cycle near-peak values of the timevarying $\mathrm{V}$ and $\mathrm{I}$ (eight for $\mathrm{V}$, eight for $\mathrm{I}$ ), several nodes for the "static" weld parameters, and three node groups arranged as a sort of block shift register, each of the blocks being characterized by a successively longer integration time than its neighbor (and its signal source). "Previous-cycle" hidden layer outputs were mapped onto the nodes of the first of these three groups and subsequently shifted leftward one block per weld half-cycle. This network proved surprisingly adept at classifying weld quality as long as training data and test data were acquired with the same welding apparatus. Moreover, such a network on several occasions correctly assessed a weld that a human weld assessor had misclassified. Perhaps most intriguing is the fact that the attractor networks demonstrated a capability for recovering from initial error. Thus, the output response for a weld ultimately properly rated as "good" could, if welding conditions were initially ambiguous, swing in the opposite direction for several cycles before moving toward, and finally to, the proper classification endpoint (attractor). Those welds which properly fell along the boundary between good and bad led to oscillatory network responses with network output never moving far from half full output value.

Figure 2 illustrates trained network responses for a series of 30 welds of various durations. Of these, one tenth were previously unseen by the network. Although overall classification accuracy was of the same order as that exhibited by the static network system described in the next section (95-98 percent), the capability conferred by the attractor network scheme for dealing with dynamic systems would appear to confer advantage in those cases for which "realtime" operation was required.

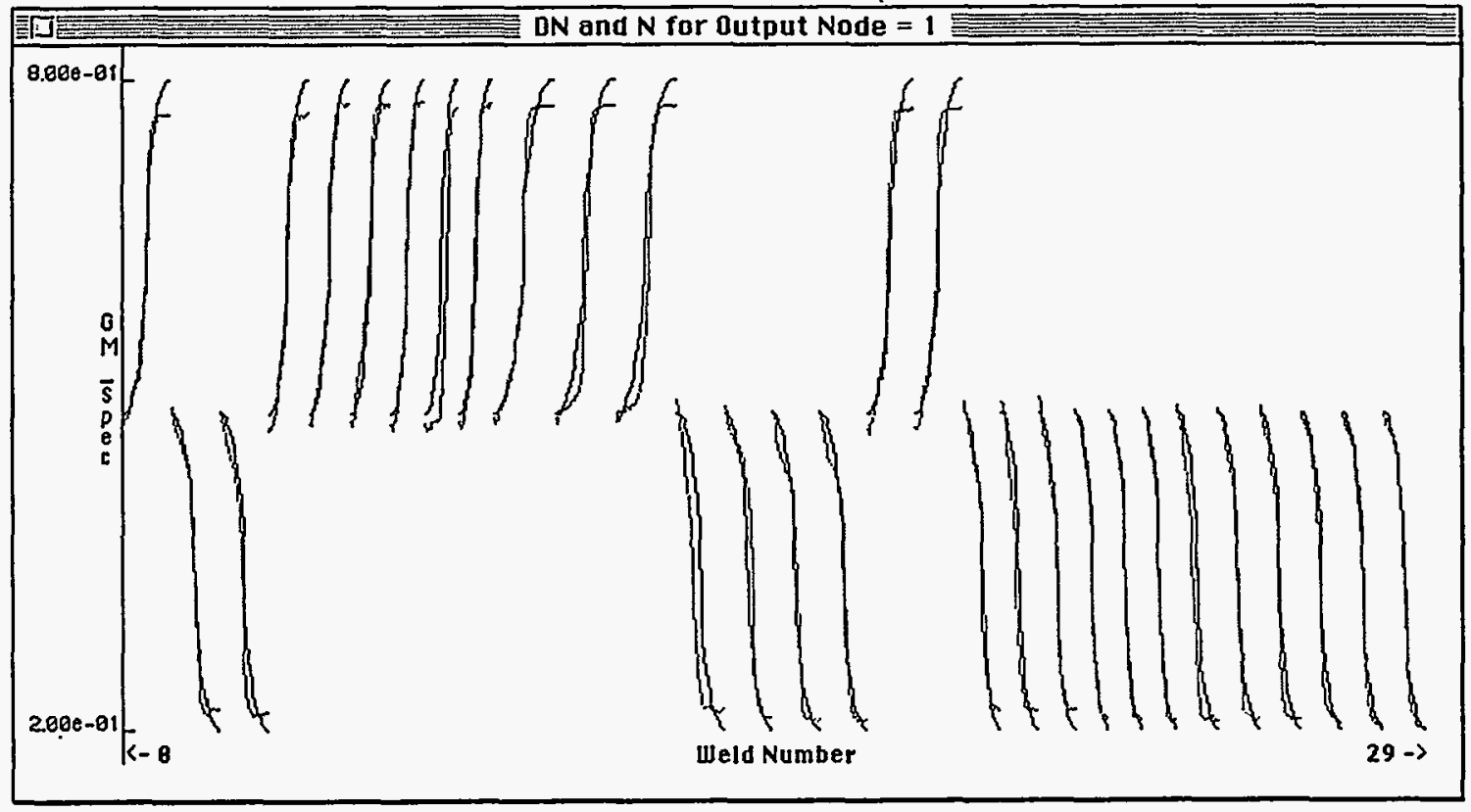

Fig. 2. Weld quality prediction with recurrent network 


\section{WELD QUALITY ASSESSMENT WITH STATIC NEURAL NETWORKS}

The development of the static network method for weld quality assessment arose as a result of an attempt to develop software that would exhibit minimum sensitivity to the effects of significant, but not quantified, variability of welding process parameters. This section covers data analysis, neural network design, and results obtained in using the static neural network classifier. Two experimental designs, the second narrower in focus than the first, define the scope of the presented material. Figure 3 is a high-level representation of the components comprising the static weld classification system.
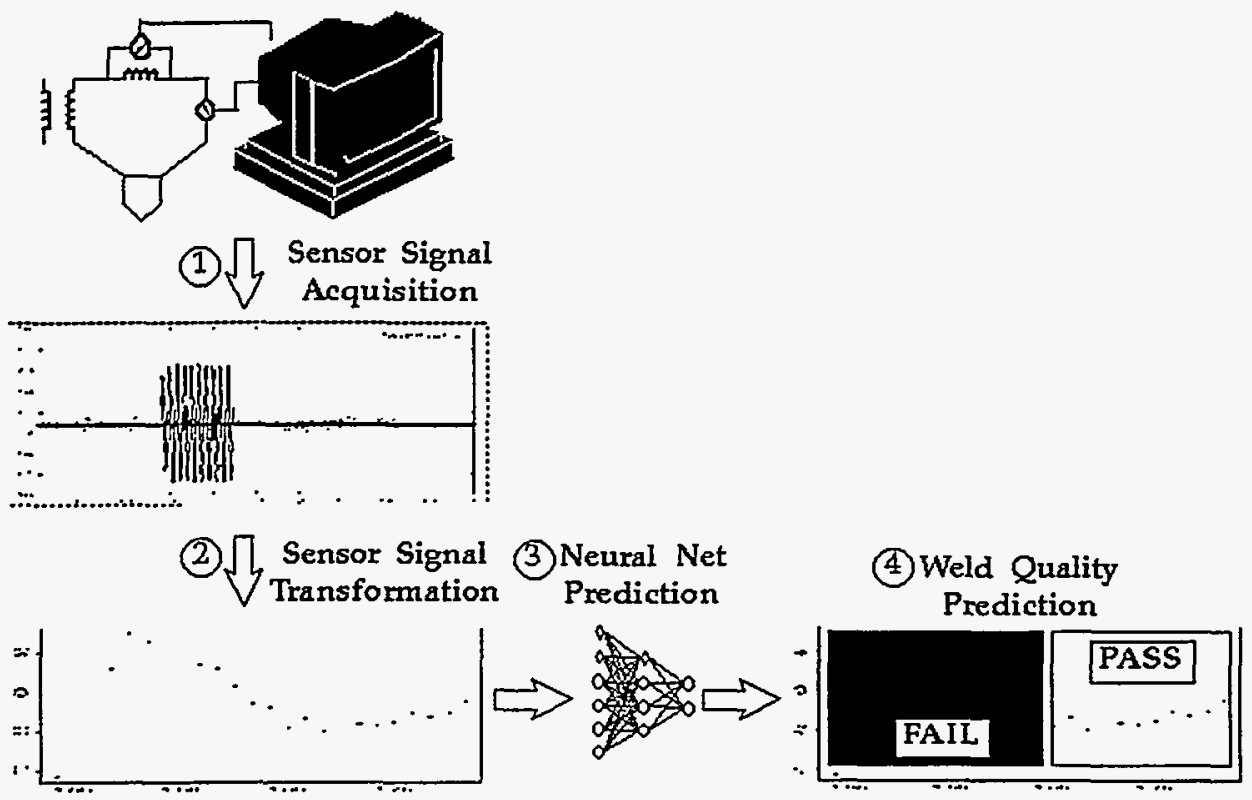

Fig. 3. Functional representation of "static" weld quality prediction system

\section{Preliminary Data Analysis and Neural Network Design}

Data analysis for the static network system was performed in the context of three time-varying parameters, current through the secondary winding of the welder transformer (I), welder transformer secondary voltage (V), and the voltage appearing at the previously noted "compensation" coil (Vc). An exploratory data analysis performed on a number of other process parameters (e.g., SCR voltage, SCR firing angle, power factor) suggested that the information content of these variables was not significant.

In order to represent the time dependent $\mathrm{I}, \mathrm{V}$, and $\mathrm{Vc}$ values to the static network, these data were unfolded into the components of a static, multi-block, input vector of which each block represented data for a single weld half cycle. Desired outputs of the network classifier were taken to represent "PASS" and "FAIL" values for weld quality. It should be noted, however, that the network was not forced to learn the mapping of weld data to weld quality directly. Instead, the classifier system was so constructed that its two outputs computed the values of the two attributes of the spot weld on which classification is traditionally based, the nugget size (NS) and the indentation value (Ind). The computed values for NS and Ind were employed, not directly in a classification formula, but to identify "updated" threshold values in a weld classification algorithm.

The rationale for this treatment of network output followed from the requirement to manage the variations in unobserved parameters in an optimal manner with respect to the classification task. This requirement arose in turn from the earlier established fact that the information content of the measured variables (I, V, and Vc) did not appear sufficient to lead to construction of a static network-based classifier of the desired accuracy. This forced a trade-off among classifier characteristics. In particular, it was deemed better to be able to classify correctly all failing welds at the expense of incorrectly classifying some of the good welds as failing rather than to risk mis-identification of failing welds. Network-computed values for NS and Ind supported a classification threshold update scheme by which this trade-off was effected. 


\section{Experimental Design \#1}

The defining condition for the first experimental design was to maintain a realistic level of difficulty for the classification task in the context of a reduced, yet significant, variation in welding schedules. Controlled variables were limited to Tip Force, Number of Weld Cycles, Weld Current, and Upslope Current. A training data set of 144 experimental welds divided into three groups was created. The groups were defined by Tip Force values of 400 , 500 , and $600 \mathrm{lb}$., respectively. Within each of these three groups, the Number of Weld Cycles was limited to the values 4, 6, and 8. Finally, for each fixed Number of Weld Cycles within each of the three groups, both Upslope Current and Weld Current were varied simultaneously to obtain 16 different combinations. Sixteen additional welds were created for use as testing data. Parameters for this group of welds were intentionally chosen as duplicates of those of members of a previous subgroup.

\section{Analysis of Experimental Results \#1}

The primary purpose of the experiment \#1 data analysis was to identify a "signature" of the welding process that would allow reliable classification of resistance spot welds. Two important points emerged: (1), most process parameters (e.g., Power Factor, Firing Angle) did not appear to contain discriminating information relevant to the classification task; and (2), the raw, unprocessed input signals $(\mathrm{V}, \mathrm{I}$, and $\mathrm{Vc}$ ) were not themselves sufficiently discriminating for the static network-based classification task.

At this juncture, use was made of previous work on the Dynamic Resistance Curve [1] in an attempt to construct a representation more suitable for the classification task. The important result here was that the raw input signals (for $\mathrm{V}$ and $\mathrm{l}$ ), when transformed into the points on the dynamic resistance curve for the welding process clearly contained discriminating information. The nature of the signal acquisition process employed for the present studies suggested that a compensation representation of the form given by Eq. 1 would recover the approximate form of the dynamic resistance for $k$ equal 5.0.

$$
\mathrm{R}=\left(\mathrm{V}-\mathrm{k}^{*} \mathrm{Vc}\right) / \mathrm{I} \text {, }
$$

Neural Network Classifier Design for Experimental Results \#1

The architecture for the first static network-based classifier was a simple Perceptron comprising an input layer, a single hidden layer, and an output layer. The number of input units in a specific network depended on the number of weld cycles in the experimental group with which that network was associated. For example, the second group of weld experiments was defined by 6 Up Slope cycles and 6 Weld cycles. Thus, the associated network included 24 input units (two units for each complete AC cycle, or one per so-called "weld half-cycle"). The signal represented by each of these units was taken as the value of the "dynamic resistance" computed according to Eq.1. Two output units were included in all static classification networks, one for computing the value of the Nugget Size, the other for computing the Indentation value. Training for these networks involved a form of cross-validation wherein the complete data set for any one experimental group was divided into variously partitioned training and testing sets.

Although the classification accuracy observed in the cross-validation tests was observed to be in excess of $80 \%$, the performance of the classifier was relatively poor in those cases for which the welds were either marginally passing or marginally failing. The experiment described in the next section was designed to investigate whether classification performance could be improved for such marginal cases.

\section{Experimental Design \#2}

For the second experiment, the number of controlled variables whose values were varied was further constrained. The intent was to investigate whether classifier accuracy would increase given training cases covering a limited subspace of the welding process parameters.

Data for the second experiment comprised 96 training instances divided into two groups and 16 testing instances. The Tip Force was held constant at $500 \mathrm{lb}$. and the Welding Cycle value was fixed at 6 . The only variable parameters were the Upslope Current and the Welding Current. For the training set, these were varied to obtain 16 combinations. For each of these combinations, three experimental welds were generated so as to capture the effect of the unobservable variables. Each of the first two groups of experimental welds thus bore $16 \times 3=48$ welds. The third testing group included a single weld for each of the 16 Upslope Current/Welding Current combinations. 


\begin{abstract}
Analysis of Experimental Results \#2
Data analysis for the second experiment confirmed the previous finding that there is a significant correlation between the data points transformed so as to represent a dynamic resistance curve and the change in values of Indentation and Nugget Size variables. However, it was also apparent that, in the borderline cases for which the welds were either marginally passing or marginally failing, the constructed dynamic resistance curves did not exhibit visible discriminating characteristics. The task was now to determine whether a neural network classifier could extract features that were not obvious from visual inspection of these data points.
\end{abstract}

Neural Network Classifier Design for Experimental Results \#2

The neural network architecture for the second set of experiments was identical to that employed in the previous stage. Here, however, a "power" representation of weld parameters (Eq. 2, below), rather than the expression of Eq. 1 , was used to represent the dynamic resistance curve points to the network classifier.

$$
\mathrm{P}=\left(\mathrm{V}-\mathrm{k}^{*} \mathrm{Vc}\right) * \mathrm{I}
$$

Results of network training involving the power representation of dynamic resistance were substantially improved relative to those of the earlier employed "static" schemes. In the cross-validation tests, the classification performance was now observed to be in excess of $95 \%$. When the constructed network classifier was tested on the cases from the previous experimental design \#1, the same result was observed. This held true irrespective of the fact that the Tip Force in the experimental run \#1 was in some cases different from that employed in the present experiment. Parallel validation experiments were performed in the laboratory and welding shop environments with similar results.

\title{
CONCLUDING REMARKS
}

The studies reported here offer clear evidence that relatively simply obtained resistance spot welding data embody signatures that can be extracted and employed for weld quality assessment. The observed $95 \%$ classification accuracy for a series of experimental runs performed at significantly different times and for different process parameters suggests considerable potential for the static network method. The dynamic method, although less extensively studied, appears to offer considerable potential in applications for which the ability to perform classifications during the evolution of a physical process is of importance.

At the most general level, we assert that we have demonstrated that neural networks can be employed to address two significant problems confronting the resistance spot welding industry, control of the process itself, and both real-time and rapid a posteriori determination of the quality of completed welds. The primary objective of the future work is to perform experimental and modeling studies to validate the neural network technology and determine if it is appropriate for quality assessment within a broader industrial context. The main task to be completed is evaluation of the sensitivity and robustness of the developed neural network-based approaches in such a context.

\section{ACKNOWLEDGMENTS}

This research was sponsored by the Office of Basic Energy Sciences, Division of Materials Science, U.S. Department of Energy, under contract DE-AC05-96OR22464 with Lockheed Martin Energy Research Corporation. This submitted manuscript has been authored by a contractor of the U. S. Government under Contract No. DE-AC05960R22464. Accordingly, the U. S. Government retains a non-exclusive, royalty-free license to publish or reproduce the published form of this contribution, or allow others to do so, for U. S. Government purposes.

\section{REFERENCES}

1. S. A. Gedeon, C. D. Sorensen, K. T. Ulrich, and T. W. Eagar, 1987. Measurement of Dynamic Electrical and Mechanical Properties of Resistance Spot Welds. Welding Journal, December 1987, 378-385. 